\title{
The insertion of a percutaneous endoscopic sigmoidostomy tube
}

\author{
V. $\operatorname{Cubas}^{1} \cdot$ O. Adedeji ${ }^{1}$
}

Received: 28 February 2016/Accepted: 27 April 2016/Published online: 10 May 2016

(C) Springer-Verlag Italia Srl 2016

We present a video demonstration of insertion of a percutaneous endoscopic sigmoidostomy tube and a brief overview of possible problems and aftercare.

The main indications are recurrent sigmoid volvulus and chronic pseudo-obstruction. It may also be used for chronic constipation to administer enemas.

It is a safe procedure with minimal morbidity for commonly encountered problems often necessitating repeat hospitalisation.

Percutaneous endoscopic sigmoidostomy offers an alternative treatment for patients who have tried conventional treatment options without success. Traditional treatment options for sigmoid volvulus and pseudo-obstruction comprise endoscopic decompression and/or open resection. However, these management options have varying success with endoscopic decompression having a recurrence rate of approximately $40 \%$, and open resection may be contraindicated for frail, elderly patients or the severely immunocompromised.

\section{Compliance with ethical standards}

Conflict of interest The authors declare that they have no conflict of interest.

Ethical approval Formal ethical approval application not required for this video presentation.

Informed consent Written informed consent was acquired for the making of this educational video.
Electronic supplementary material The online version of this article (doi:10.1007/s10151-016-1476-9) contains supplementary material, which is available to authorized users.

\section{Cubas}

vanessacubas@nhs.net

1 University Hospital Birmingham, Mindelsohn Way, Edgbaston, Birmingham B15 2TH, UK 